tem by assuming that bacteriophage $\phi 6$ has evolved to reach the fittest genome to replicate in its host whereas human influenza virus has not. Because the fittest flu genome has yet not evolved, the accumulation of mutations as a consequence of population bottlenecks yields a more fit genome. In other words, the ratchet operates only on genomes that have reached maximum fitness.

RUBEN O. DONIS Department of Veterinary Science, University of Nebraska,

Lincoln, Nebraska 68583, USA

\section{Sexual semantics}

SIR - J. L. Martinez (Nature 352, 288; 1991) objects to the use of the term 'sex' to describe the diverse recombination processes in bacteria (J. Maynard Smith et al. Nature 349, 29-31; 1991), preferring to reserve the term for "the well-characterized mechanism of reproduction-linked genetic recombination in eukaryotes". But if bacteria don't have sex, neither do many eukaryotes.

Many eukaryotes are protists, many of whom reproduce without sex, have sex without reproducing, and have sex with themselves. To assume that recombination in bacteria is "completely unrelated to what we call sex" is to ignore the fact that protists (and bacteria) tell us that reproduction, reduction division, crossing-over, ploidy and syngamy are separate phenomena, which may or may not have evolved under similar selective forces. This is precisely why these groups are so interesting, and why we should adopt a liberal attitude towards 'sex'.

INNES C. CUTHILL

Department of Zoology,

University of Bristol,

Bristol BS8 1UG, UK

SIR - Martinez ${ }^{1}$ doubts whether it is appropriate to use the term 'sex' in the context of bacteria and would restrict its use to "the well-characterized mechanism of reproduction-linked genetic recombination in eurkaryotes, leaving bacteria with their own very different mechanisms of genetic recombination which are usually completely unrelated to what we usually call sex".

Precisely this problem beset us when compiling entries for a biology dictionary $^{2}$. We hope we were helpful when we wrote the entry for sexual reproduction (capitals indicate crossreferences), which begins as follows: "In its broadest sense, any process by which genetic material is transferred from one cell, and/or individual, to another (see CONJUGATION); or any process involving genetic RECOMBINATION. Such a view of sexual reproduction would have to include PLASMID trans- fer, viral infection, antibody production and even some forms of GENE MANIPULATION. One possible benefit of throwing the concept wide open this way is that it may reveal possible clues to the origin of SEX. The processes of sex (recombination and/or genetic transfer) and reproduction (production of new individuals) are sometimes only tenuously linked (e.g. in PARTHENOGENESIS in Paramecium), and may even be uncoupled

In the entry on SEX we point out that this term is often used as a synonym of sexual reproduction, and attempt to distinguish its several other senses most of which involve organisms where different sexes or sex organs are identifiable.

We welcome suggestions for improvements to these and other entries.

Harrow School,

Michael THAin

Harrow,

Middlesex HA1 3HW, UK

1. Martinez, J. L. Nature 352, 288 (1991)

2. Abercrombie, M., Hickman, M., Johnson, M. L. \& Thain, $\mathrm{M}$. The New Penguin Dictionary of Biology (Penguin, London 1990).

\section{Ocean uncertainty}

SIR - Numerical climate models typically predict that doubling atmospheric $\mathrm{CO}_{2}$ would lead to eventual, or equilibrium, global warming of $2-5 \mathrm{~K}$. How quickly the warming would occur depends in part on the behaviour of the oceans, which influence the rate of greenhouse warming by taking up both heat and $\mathrm{CO}_{2}$ from the atmosphere. Considering the heat-uptake question alone, studies with coupled oceanicatmospheric general circulation models make it clear that the rate at which the climate would change is modeldependent to an even greater extent than is the equilibrium warming. Further, a recent simulation suggests that much of the uncertainty in rate of climatic change may be due to uncertainty in oceanic rather than atmospheric processes.

The table shows lag times derived from published results of coupled oceanic-atmospheric general circulation models, each of which was subjected to a sudden step-function doubling of atmospheric $\mathrm{CO}_{2}$. Although the lag time of the real climate system depends on the history of greenhouse gas concentrations, the idealized case of sudden $\mathrm{CO}_{2}$ doubling provides an upper limit to the spread of model response times under a common scheme of greenhouse forcing. In the table, $\Delta T_{\text {eq }}$ denotes the equilibrium surface air warming due to doubled $\mathrm{CO}_{2}$, and $\tau_{e}$ denotes the e-folding relaxation time - the time it takes after $\mathrm{CO}_{2}$ is doubled to reach
MODEL RESPONSES TO DOUBLED $\mathrm{CO}_{2}$

Model

Ref.

$\underset{(\mathrm{K})}{\Delta T_{\mathrm{eq}}}$

$\tau_{\mathrm{e}}$
$(\mathrm{yr})$

$\left(\begin{array}{c}K_{\text {eff }} \\ \left(\mathrm{cm}^{2} \mathrm{~s}^{-1}\right)\end{array}\right.$

GISS

OSU

NCAR

GFDL

OPYC

5
6
7
8
9

4.2

2.8

124

2.6

$3.5 ?$

2.4

46

2.1

1.5 ?

+ ECHAM

Question mark, uncertainty about whether equilibrium was reached.

$1-e^{-1} \approx 63 \%$ of $\Delta T_{\text {eq }}$. Among this set of models, $\Delta T_{\text {eq }}$ varies by less than a factor of 2 , but $\tau_{\mathrm{e}}$ varies by nearly an order of magnitude.

The right-hand column of the table is an attempt to identify the part of the uncertainty in lag time due to the oceanic component of the coupled models. As shown by Hansen et al. ${ }^{1}$ and Wigley and Schlesinger ${ }^{2}$, if downward heat flux in the oceans is proportional to the vertical temperature gradient, so that the ocean in effect transports heat by diffusion, then $\tau_{\mathrm{e}}$ scales as $\left(\Delta T_{\text {eq }}\right)^{2}$. To separate out this $\Delta T_{\mathrm{eq}}$-dependence, the right-hand column gives the effective thermal diffusivity $\kappa_{\text {eff }}$ of the oceans according to Wigley and Schlesinger's analytical model ${ }^{2}: \tau_{\mathrm{e}} \approx\left(2.74 \mathrm{~cm}^{-2} \mathrm{~s} \mathrm{~K}^{-1}\right)$ $\left(\kappa_{\text {eff }}\right)\left(\Delta T_{\text {eq }}\right)^{2}$. If the analytical ocean model is modified to account for heat transport due to the sinking of cold water at the poles (thermohaline circulation), the dependence of $\tau_{\mathrm{e}}$ on $\Delta T_{\mathrm{eq}}$ is less steep than second power ${ }^{3}$, so the range of uncertainty in oceanic processes may be underestimated by the range of $\kappa_{\text {eff }}$ among the models.

I find that $\kappa_{\text {eff }}$ varies by less than a factor of two among the GISS, NCAR, OSU and GFDL simulations, suggesting that most of the variation in $\tau_{\mathrm{e}}$ among these models is actually due to variations in $\Delta T_{\text {eq }}$. The GISS simulation uses a simplified, purely diffusive ocean model whereas the remaining three use Cartesian-coordinate oceanic general circulation models of the type developed two decades ago at GFDL. But the final entry, a simulation with the Hamburg version of the ECMWF atmospheric model coupled with a new 'isopycnic' oceanic model developed by J. M. Oberhuber, obtains a significantly faster ocean response than the other models even if it is assumed that $\tau_{\mathrm{e}}$ scales as $\left(\Delta T_{\text {eq }}\right)^{2}$. Although this particular result is highly preliminary, the quick surface warming may be attributable to the careful restriction of heat diffusion across surfaces of constant density in Oberhuber's model, which alone of all the ocean models shown in the table uses density as a vertical coordinate.

Narrowing uncertainty in the rate of climatic change is important for compar- 